\title{
Reducing tau aggregates with anle138b delays disease progression in a mouse model of tauopathies
}

\author{
Jens Wagner ${ }^{1} \cdot$ Sybille Krauss $^{1} \cdot$ Song Shi $^{2} \cdot$ Sergey Ryazanov $^{3,4} \cdot$ Julia Steffen $^{1} \cdot$ \\ Carolin Miklitz $^{1}$ - Andrei Leonov ${ }^{3,4,5}$ - Alexander Kleinknecht ${ }^{6} \cdot$ Bettina Göricke $^{6}$. \\ Jochen H. Weishaupt ${ }^{6} \cdot$ Daniel Weckbecker $^{5} \cdot$ Anne M. Reiner $^{7} \cdot$ Wolfgang Zinth ${ }^{7}$. \\ Johannes Levin $^{8} \cdot$ Dan Ehninger $^{1} \cdot$ Stefan Remy $^{1} \cdot$ Hans A. Kretzschmar ${ }^{2}$. \\ Christian Griesinger ${ }^{3,4,5}$ - Armin Giese ${ }^{2,5} \cdot$ Martin Fuhrmann $^{1}$
}

Received: 24 July 2015 / Revised: 24 September 2015 / Accepted: 24 September 2015 / Published online: 6 October 2015

(C) Springer-Verlag Berlin Heidelberg 2015

\begin{abstract}
Pathological tau aggregation leads to filamentous tau inclusions and characterizes neurodegenerative tauopathies such as Alzheimer's disease and frontotemporal dementia and parkinsonism linked to chromosome 17. Tau aggregation coincides with clinical symptoms and is thought to mediate neurodegeneration. Transgenic mice overexpressing mutant human P301S tau exhibit many neuropathological features of human tauopathies including behavioral deficits and increased mortality. Here, we show that the di-phenyl-pyrazole anle138b binds to aggregated
\end{abstract}

Electronic supplementary material The online version of this article (doi:10.1007/s00401-015-1483-3) contains supplementary material, which is available to authorized users.

Martin Fuhrmann

martin.fuhrmann@dzne.de

1 German Center for Neurodegenerative Diseases (DZNE), Ludwig-Erhard-Allee 2, 53175 Bonn, Germany

2 Center for Neuropathology and Prion Research, Ludwig-Maximilians-Universität, Feodor-Lynen-Str. 23, 81377 Munich, Germany

3 NMR Based Structural Biology, Max Planck Institute for Biophysical Chemistry, Am Fassberg 11, 37077 Göttingen, Germany

4 DFG Center for Nanoscale Microscopy and Molecular Physiology of the Brain (CNMPB), 37073 Göttingen, Germany

5 MODAG GmbH, 55324 Wendelsheim, Germany

6 Neurologie, Universitätsmedizin Göttingen, 37075 Göttingen, Germany

7 BioMolekulare Optik, Ludwig-Maximilians-Universität, 80538 Munich, Germany

8 Neurologische Klinik, Klinikum der Ludwig-MaximiliansUniversität, Munich, Germany tau and inhibits tau aggregation in vitro and in vivo. Furthermore, anle138b treatment effectively ameliorates disease symptoms, increases survival time and improves cognition of tau transgenic PS19 mice. In addition, we found decreased synapse and neuron loss accompanied by a decreased gliosis in the hippocampus. Our results suggest that reducing tau aggregates with anle $138 \mathrm{~b}$ may represent an effective and promising approach for the treatment of human tauopathies.

Keywords Alzheimer's disease · Tauopathy · Tau · Protein aggregation $\cdot$ Anle138b $\cdot$ Tau aggregation inhibitor

\section{Introduction}

The conversion of soluble tau monomers into insoluble fibrils is a key component in the pathogenesis of neurodegenerative tauopathies, including Alzheimer's disease, frontotemporal dementia and parkinsonism linked to chromosome 17, Pick's disease, progressive supranuclear palsy, and corticobasal degeneration [30, 44]. In Alzheimer's disease, tau pathology correlates with neuronal loss and clinical symptoms $[2,10,18]$. In experimental mouse models, accumulation of aggregated tau is associated with memory deficits and neurodegeneration [6, 40, 41]. Inhibiting the aggregation of tau with small molecules reduced neurotoxicity in vitro [29]. Together, these observations suggest that aggregated tau is not merely a neuropathological marker; tau aggregation rather represents a main target of a diseasemodifying therapy for tauopathies.

At present, no disease-modifying treatment for tauopathies exists [25, 44]. Despite the identification of tau aggregation inhibitors in vitro [4, 13, 38, 39, 48], only methylene blue was tested in a tau transgenic mouse model and 
clinical trials are underway. However, methylene blue has several targets $[1,16,20,36,37]$. Therefore, the therapeutic effects and reduction of tau aggregates may not be exclusively based on protein aggregation inhibition. Thus, it is not clear whether inhibition of tau aggregation is beneficial in mouse models of tauopathy. Here, we test a new compound with high bioavailability and low toxicity [47] with regard to tau aggregation inhibition and therapeutic efficacy in a tau transgenic mouse model.

Previously, it has been shown that the di-phenyl-pyrazole anle138b [5-(1,3-benzodioxol-5-yl)-3-(3-bromophenyl)$1 H$-pyrazole] acts as an oligomer modulator of $\alpha$-synuclein and prion protein. Anle138b delays disease progression in mouse models of both prion disease and Parkinson's disease by targeting pathological aggregates of $\alpha$-synuclein and prion protein [47]. A characteristic of several aggregation inhibitors represents their ability to block the pathological aggregation of other proteins like $A \beta$ and $\alpha$-synuclein [34]. Based on this, we hypothesized that anle138b could also reduce pathological tau aggregates in vivo and delay disease progression in a mouse model of tauopathies.

We chose transgenic PS19 mice overexpressing mutant human tau (P301S) [50]. In humans, the P301S mutation causes familial frontotemporal dementia [12, 43]. This well-characterized mouse model displays the identified neuropathological hallmarks of human tauopathies, pathological behavioral phenotypes, and increased mortality $[11,50]$. We administered anle $138 \mathrm{~b}$ orally from weaning to terminal disease, and examined both pathogenesis and survival. Non-transgenic littermates (ntg) were likewise treated and used as controls for potential side effects of anle138b.

\section{Materials and methods}

\section{Animals and treatment}

PS19 human tau transgenic mice [50] were obtained from The Jackson Laboratories (Stock number 8169, The Jackson Laboratory) and group housed separated by gender in individually ventilated cages (IVCs) under specified pathogen-free (SPF) conditions. Age- and sex-matched nontransgenic littermates were used for all experiments and equal numbers of male and female mice were randomly assigned to the experimental groups. Mice had unlimited access to food and water, the light and dark cycle was $12 \mathrm{~h} / 12 \mathrm{~h}$ and the temperature was kept constant at $22{ }^{\circ} \mathrm{C}$. The age of mice was 10 to 12 months for biochemical and immunohistochemical analysis. For cognitive performance test, mice were 9 months of age. The compound anle138b was administered with drug-supplemented food pellets ( $2 \mathrm{~g}$ compound/kg food; Ssniff, Soest, Germany).
The compound was added during the manufacturing process of the food pellets as dry powder without a vehicle. Therefore, control mice were treated with non-supplemented food pellets. The treatment was initiated after weaning and lasted until the time of kill. Animals were monitored daily for clinical signs by trained animal caretakers blinded to the study design and body weight was assessed monthly. The animals were killed, when they had reached the terminal stage of disease. From all animals, we collected one brain hemisphere, which was immediately frozen at $-80{ }^{\circ} \mathrm{C}$, for biochemical analysis and the other hemisphere after fixation in $4 \%$ paraformaldehyde for histological analysis. The experimental procedures were in accordance with guidelines established by the DZNE and were approved by the government of North-Rhine-Westphalia.

\section{Protein purification and aggregation}

Tau isoform hTau46 (1N4R) was expressed from a pRK172 vector in BL21(DE3) E. coli cells. The cells were grown at $37{ }^{\circ} \mathrm{C}$ in $\mathrm{LB}$ medium containing $100 \mu \mathrm{g} /$ $\mathrm{mL}$ ampicillin. Protein expression was induced by addition of $1 \mathrm{mM}$ isopropyl-1-thio- $\beta$-D-galactopyranoside. After $3 \mathrm{~h}$ of incubation cells were harvested by centrifugation, resuspended in $50 \mathrm{mM} \mathrm{NaP}_{\mathrm{i}} \mathrm{pH} 7.0$ containing $1 \mathrm{mM}$ EGTA and $1 \mathrm{mM}$ DTT, and lysed by boiling for $30 \mathrm{~min}$. To remove cell debris, the lysate was centrifuged for $15 \mathrm{~min}$ at $14,600 \mathrm{rpm}$ and $4{ }^{\circ} \mathrm{C}$ in a Th-641 rotor. The supernatant was filtered through a $45-\mu \mathrm{m}$ filter and loaded onto a GE HiTrap SP HP column $(5 \mathrm{~mL})$. The bound protein was eluted with a salt gradient from 0 to $300 \mathrm{mM} \mathrm{NaCl}$. Elution fractions containing hTau46 were pooled and loaded onto PD-10 desalting columns. hTau46 was eluted in $50 \mathrm{mM}$ Tris $\mathrm{pH} 7.0$ and the protein concentration was determined via a BCA assay. The eluate was collected in aliquots, frozen in liquid nitrogen and stored at $-80{ }^{\circ} \mathrm{C}$. For aggregation, frozen hTau 46 was thawed at RT and incubated in $50 \mathrm{mM}$ Tris $\mathrm{pH} 7.0$ containing $0.02 \% \mathrm{NaN}_{3}$ and $0.03 \mathrm{mg} / \mathrm{ml}$ heparin sodium salt (Sigma Aldrich, Switzerland) for $72 \mathrm{~h}$ at $37{ }^{\circ} \mathrm{C}$ under constant agitation (1000 rpm). Aggregated hTau46 was frozen in liquid nitrogen and stored at $-80{ }^{\circ} \mathrm{C}$. The monomeric and aggregated protein was thawed at RT directly before the fluorescence measurements.

\section{Anle138b fluorescence measurements}

All fluorescence measurements were performed with a spectrofluorometer (Horiba, Fluorolog3) at $300 \mathrm{~nm}$ excitation wavelength (power: $0.9 \mu \mathrm{W}$, front face geometry) in combination with a home build stirring system, as described previously [47]. In brief, two solutions of 
anle138b (250 nM) were prepared in buffer (containing $0.0025 \%$ DMSO). The first solution (S1) was obtained by adding $5 \mu \mathrm{l}$ of a $10 \mathrm{mM}$ anle138b solution in DMSO to $200 \mathrm{ml}$ buffer ( $50 \mathrm{mM}$ Tris buffer $\mathrm{pH}$ 7.0). To stabilize aggregated hTau46 the second solution (S2) was prepared by adding additionally $0.02 \% \mathrm{NaN}_{3}$ and $0.03 \mathrm{mg} /$ $\mathrm{mL}$ heparin sodium salt (Sigma). Both solutions were stirred under ultra-sound for $10 \mathrm{~min}$ at about $30^{\circ} \mathrm{C}$. Two fused silica cells (Hellma, $10 \times 4 \mathrm{~mm}$, Typ 117.104FQS) were filled with solutions S1 and S2 and fluorescence spectra were recorded. Every $15 \mathrm{~min}$ monomeric (from a $22 \mu \mathrm{M}$ stock solution) and aggregated $(9.3 \mu \mathrm{M}$ stock solution) hTau46 was added to cells $\mathrm{S} 1$ and $\mathrm{S} 2$, respectively. The volumes of added stock solution were adjusted to yield the concentrations given in (Fig. 1a). Fluorescence measurements were performed for both cells after each protein addition step. To correct for the small emission of tyrosine of the hTau46, the fluorescence spectra of two reference cells containing the same amount of monomeric and aggregated hTau 46 as well as the same buffer solutions but no anle138b were subtracted. All data have been smoothed via moving average over five points.

\section{In vitro tau aggregation assay}

The human tau isoform hTau46 (1N4R) was expressed in E. coli BL 21 (DE3) RIL and subsequently purified as described previously [3]. To prepare tau protein for confocal single molecule measurements, it was labeled with fluorescent dyes Alexa488-O-succinimidyl-ester or Alexa647$O$-succinimidyl-ester (Invitrogen, Darmstadt, Germany) as described. Fluorescently labeled tau was mixed as a tenfold stock solution in $50 \mathrm{mM}$ tris buffer, $\mathrm{pH}$ 7.0. The stock solution was adjusted to a final ratio of tau- 488 to tau- 647 of approximately $1: 1$. The final concentration of labeled tau in the aggregation assay was between 10 and $20 \mathrm{nM}$ in a total sample volume of $20 \mu \mathrm{l}$. Before each aggregation experiment, preformed oligomers were removed by ultracentrifugation, and the stock solution was scanned for pre-existing aggregates by SIFT. Only samples free of preformed aggregates were used.

The tau aggregation assay was conducted with minor modifications as published previously for an $\alpha$-synuclein aggregation assay [47]. In brief, experiments were performed in $50 \mathrm{mM}$ Tris buffer $(\mathrm{pH} 7.0)$ in a total volume of $20 \mu \mathrm{l}$ in 384-well plates with a cover slide, bottom
Fig. 1 Anle138b binds to aggregated tau and reduces tau aggregates in vitro. a Fluorescence spectrometry of anle $138 \mathrm{~b}$ $(250 \mathrm{nM})$ excited at $300 \mathrm{~nm}$ with tau monomers (left panel) and pre-aggregated tau protein (right panel). Tau monomers did not change the fluorescence spectra of anle $138 \mathrm{~b}$, whereas addition of pre-aggregated tau protein changed the fluorescence properties of anle138b and increased fluorescence at $340 \mathrm{~nm}$. b Exemplary SIFT measurement (SIFT scanning for intensely fluorescent targets). $\mathbf{c}$ The formation of tau aggregates in vitro is significantly inhibited by the application of anle138b $(p<0.05, t$ test). d Correlation between tau anti-aggregative effect and compound brain concentration. Anle $138 \mathrm{~b}$ shows the best correlation of tau aggregation inhibition and bioavailability
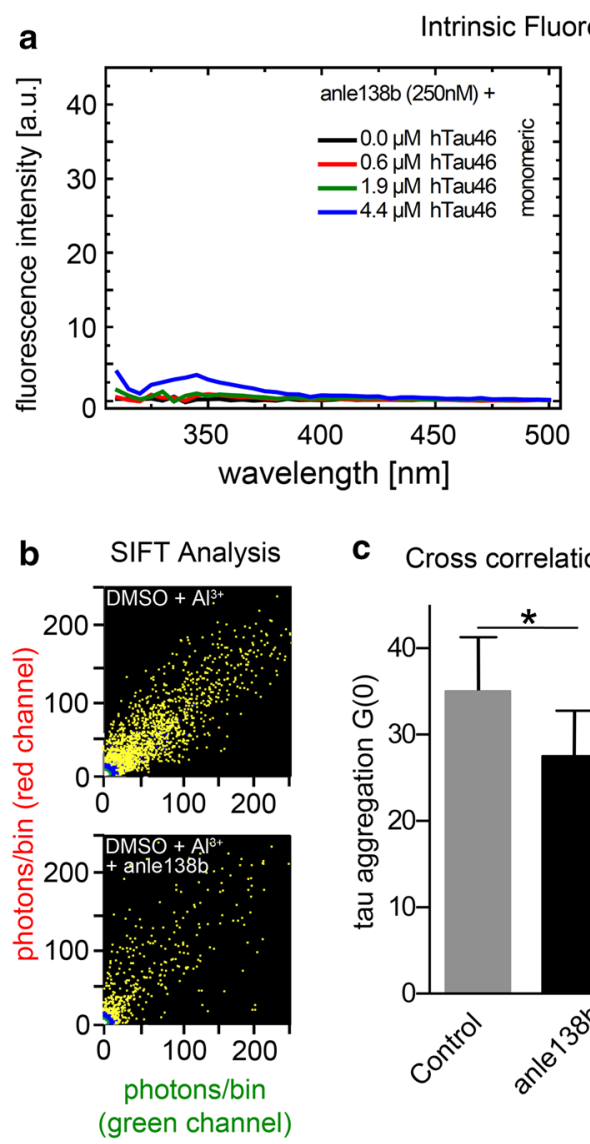

C Cross correlation

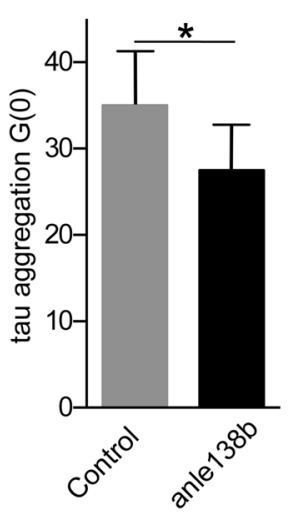


(Greiner Bio-One, Germany). Plates were covered with adhesive film to obviate evaporation. Tau aggregation was monitored in presence of final concentrations of $1 \%$ DMSO and $10 \mu \mathrm{M} \mathrm{AlCl}^{3}$. Compounds were diluted into the assay mixture from tenfold stock solutions containing $10 \%$ DMSO $(\mathrm{v} / \mathrm{v})$, resulting in a final concentration of $1 \%$ DMSO in all samples. Experiments were started by diluting the tenfold stock solution of fluorescently labeled Tau into a well, containing $1 \% \mathrm{DMSO}$ and $10 \mu \mathrm{M} \mathrm{AlCl}{ }^{3}$ or $1 \%$ DMSO, $10 \mu \mathrm{M} \mathrm{AlCl}^{3}$ and $10 \mu \mathrm{M}$ anle138b. Measurements were started after an incubation period of $30 \mathrm{~min}$. Aggregation was monitored at room temperature for at least $1 \mathrm{~h}$ in 5 independent samples for each experimental group by scanning for intensely fluorescent targets (SIFT) [8]. SIFT measurements were carried out on an Insight Reader (Evotec-Technologies) with dual-color excitation at 488 and $633 \mathrm{~nm}$, using a $40 \times 1.2$ numerical aperture microscope objective (Olympus, Japan) and a pinhole diameter of $70 \mu \mathrm{m}$ at Fluorescence Intensity Distribution Analysis (FIDA) setting. Excitation power was $200 \mu \mathrm{W}$ at $488 \mathrm{~nm}$ and $300 \mu \mathrm{W}$ at $633 \mathrm{~nm}$. Measurement time was $10 \mathrm{~s}$. Scanning parameters were set to $100 \mu \mathrm{m}$ scan path length, $50 \mathrm{~Hz}$ beam scanner frequency, and $2000 \mu \mathrm{m}$ positioning table movement. The fluorescence data were analyzed by SIFT analyze software (Evotec OAI, Germany) as described [19].

\section{Pharmacokinetic analysis of brain concentrations of anle138b}

Mice (C57BL6) were single housed and compounds were administrated in peanut butter. Animals were killed after $4 \mathrm{~h}$ of administration. Brains were taken out and immediately frozen in liquid nitrogen. Samples were stored at $-80^{\circ} \mathrm{C}$.

The tissues were thawed at $4{ }^{\circ} \mathrm{C}$ prior to use. It was homogenized twice in $5 \mathrm{~mL}$ of acetonitrile at maximum speed for 3 min using a homogenizer (IKA ULTRA-TURRAX Tube drive workstation, Germany). The homogenate was ultrasonicated at $30{ }^{\circ} \mathrm{C}$ for $5 \mathrm{~min}$ and centrifuged at $5000 \times g$ for $10 \mathrm{~min}$. An aliquot ( $100 \mu \mathrm{L}$ of supernatant) was injected into HPLC system. Briefly, analytical high-performance liquid chromatography (HPLC) was performed using a Waters HPLC system with a Waters 996 Photodiode Array Detector. All separations involved a mobile phase of $0.1 \%$ trifluoroacetic acid (TFA) (v/v) in water (solvent A) and $0.1 \%$ TFA in acetonitrile (solvent B). HPLC was performed using reversed-phase (RP) column Eurospher RP $18,100 \AA, 5 \mu \mathrm{m}, 250 \times 4.6 \mathrm{~mm}$ at flow rates of $1 \mathrm{~mL} / \mathrm{min}$ with a gradient of solvent B from 0 to $100 \%$ in $50 \mathrm{~min}$. The effluent was monitored for UV absorption at $260 \mathrm{~nm}$. Samples were quantified using peak area ratio of compounds to external standard.

\section{Brain tissue extracts and immunoblot analysis}

Brain tissue was weighed and homogenized in brain lysis buffer $(50 \mathrm{mM}$ Tris/HCl $\mathrm{pH} 7.4,150 \mathrm{mM} \mathrm{NaCl}, 10 \mathrm{mM}$ EDTA pH 8.0, 1 \% (v/v) NP-40, 1 \% (w/v) deoxycholate, $1 \mu \mathrm{M}$ okadaic acid, $1 \mathrm{mM}$ phenylmethylsulfonyl fluoride (PMSF), $1 \mathrm{mM}$ 4-(2-Aminoethyl)benzenesulfonyl fluoride (AEBSF), $20 \mathrm{mM}$ sodium fluoride, $5 \mathrm{mM}$ sodium vanadate, $5 \mathrm{mM}$ sodium pyrophosphate, protease inhibitor cocktail (Sigma Aldrich, Switzerland)) using the tissue homogenizer Precellys 24 (Peqlab, Erlangen, Germany). Brain homogenates were stored in aliquots at $-80{ }^{\circ} \mathrm{C}$.

Quantification of sarkosyl-insoluble tau protein was performed as described by Terwel et al. [45] with minor modifications. Briefly, brain homogenate was centrifuged at $15,0000 \times g$ for $30 \mathrm{~min}$. The resulting pellet was resuspended in extraction buffer $(10 \mathrm{mM}$ Tris- $\mathrm{HCl} \mathrm{pH}$ 7.4, $1 \mathrm{mM}$ EGTA, $0.8 \mathrm{M} \mathrm{NaCl}, 0.1 \mathrm{M}$ PMSF, $10 \%$ (w/v) sucrose) and centrifuged at $20,000 \times g$ for $20 \mathrm{~min}$. The supernatant was adjusted to $1 \%$ (w/v) sarkosyl and incubated for $1 \mathrm{~h}$ at room temperature with constant agitation. After centrifugation at $150,000 \times \mathrm{g}$ for $30 \mathrm{~min}$, the pellet was resuspended in $50 \mathrm{mM}$ Tris- $\mathrm{HCl} \mathrm{pH} 7.4(500 \mu \mathrm{L} / \mathrm{g}$ brain tissue). Sarkosyl-insoluble tau protein was detected with the tau antibodies HT7 (1:5000; ThermoScientific) and AT100 (1:1000; ThermoScientific). Insoluble tau was normalized relative to the tissue weight.

For quantification of total tau, brain homogenates were separated by SDS-Page on $10 \%$ polyacrylamide (Applichem, Darmstadt, Germany) gels and transferred to PVDF membranes (Immobilon-P, Millipore, USA). For immunoblotting, the membrane was blocked with Tropix I-Block (Applied Biosystems) and incubated with the primary antibody HT7 (1:5000; ThermoScientific) and E178 (1:5000; Abcam) at $4{ }^{\circ} \mathrm{C}$ overnight. After three washes in PBST, the membrane was incubated with a secondary antibody (goat anti-mouse-AP (1:10000); Cell Signaling). Tau protein was visualized with CDP-Star detection solution (Roche Applied Science, Germany). Immunoblots were quantified by a luminescence imaging system (Stella, Raytest, Germany) along with the AIDA software package (Raytest, Germany). The amount of total tau was normalized to GAPDH.

For quantification of phosphorylated tau, brain homogenates were separated by SDS-Page on $10 \%$ polyacrylamide (Applichem, Darmstadt, Germany) gels and transferred to PVDF membranes (Immobilon-FL, Millipore, USA). For immunoblotting, the membrane was blocked with Odyssey Blocking Buffer (TBS) (LI-COR) and incubated with the primary antibodies E178 (monoclonal Rabbit; 1:5000; Abcam), AT8 (monoclonal mouse; 1:1000; ThermoScientific) and AT180 (monoclonal mouse; 1:1000; ThermoScientific) at $4{ }^{\circ} \mathrm{C}$ overnight. After three washes in TBST, 
the membrane was incubated with fluorescently labeled secondary antibodies (IRDye 680RD goat anti-rabbit; LICOR) and (IRDye $800 \mathrm{CW}$ goat anti-mouse; LI-COR). Tau protein was visualized by scanning the membranes with an Odyssey Classic Imager (LI-COR). Immunoblots were quantified with ImageJ software package $1.50 \mathrm{~b}$ (National Institutes of Health, USA). The amount of phosphorylated tau was normalized to total tau in each lane.

\section{Discontinuous sucrose gradient}

Brain homogenates were adjusted to $1 \%$ (w/v) sarkosyl and incubated for $2 \mathrm{~h}$ at $20^{\circ} \mathrm{C}$ with constant agitation. After incubation, the homogenates were centrifuged at $5000 \times g$ for $5 \mathrm{~min}$. The supernatant was layered onto a sucrose step gradient (10-50\% sucrose; $400 \mu \mathrm{L} 10 \%$ sucrose, $20-50 \%$ sucrose $500 \mu \mathrm{L}$ each) and centrifuged for $2 \mathrm{~h}$ at $200,000 \times g$ at $20{ }^{\circ} \mathrm{C}$ in a SW45Ti rotor (Beckman Coulter) using an Optima ultracentrifuge (Beckman Coulter). The fractions $(500 \mu \mathrm{L})$ were collected from the bottom to the top. Samples were separated by SDS-Page on $8 \%$ polyacrylamide (Applichem, Darmstadt, Germany) gels and detected with the tau antibody HT7 (1:5000; ThermoScientific). The amount of total tau loaded onto the sucrose gradient was normalized to GAPDH.

\section{Histology and immunohistochemistry}

Mice were transcardially perfused with cold PBS after being deeply anesthetized. The brains were removed and weighed. From all animals one brain hemisphere was fixed in $4 \%$ paraformaldehyde for $24 \mathrm{~h}$ for histological examination. After fixation, the brain tissue was sectioned into $50 \mu \mathrm{m}$ tissue slices using a Leica VT1000S vibratome (Leica, Wetzlar, Germany) and immunohistochemistry was performed as described by Gogolla et al. [22] with minor modifications. Briefly, the sections were incubated with permeabilization solution ( $1 \%$ Triton X-100 in PBS) for $12 \mathrm{~h}$ under constant agitation. Subsequently, the slices were blocked with $20 \%$ BSA in PBS buffer for $6 \mathrm{~h}$. Then, the tissue sections were stained with the following primary antibodies in $3 \%$ BSA/PBS overnight: tau protein (AT8, $0.3 \mu \mathrm{g} / \mathrm{mL}$; AT100, $1 \mu \mathrm{g} / \mathrm{mL}$, ThermoScientific; MC1, 1:500, a generous gift from Peter Davies), neuronal marker (NeuN, $1 \mu \mathrm{g} / \mathrm{mL}$, Millipore; Nissl, 1:200, LifeTechnologies), synapses (anti-synaptophysin, 1:500, Millipore), microglia (Iba1, $1 \mu \mathrm{g} / \mathrm{mL}$, Wako), astrocytic marker (antiGFAP, $0.3 \mu \mathrm{g} / \mathrm{mL}$, LifeTechnologies), and autophagic marker (anti-LC3, 1:1000, Novus Biologicals; P62/ SQSTM1, 1:1000, Abcam). After three washes with $3 \%$ BSA/PBS, the slices were incubated with a correspondent secondary antibody in $3 \%$ BSA/PBS for $3 \mathrm{~h}$ (LifeTechnologies). Finally, the slices were mounted using fluorescence mounting medium (Dako, Hamburg, Germany) and images were acquired on an inverted Zeiss LSM700 confocal microscope and Zeiss AxioScan.Z1 (Zeiss, Germany). For Gallyas silver staining, brain tissue was fixed in $4 \%$ paraformaldehyde followed by paraffin embedding. Sections were silver impregnated following the Gallyas method to detect tau tangles. For quantitative analysis of tau pathology with the tau stains AT8, MC1 and Gallyas, we quantified the positively stained area in matched brain sections. Quantification was done with the ZEN software package (Zeiss, Germany). For quantification of neuronal loss, the NeuN-stained area of the hippocampal CA3 region was quantified in matched brain sections. For each mouse, three sections per brain were quantified. The NeuN area was analyzed using imaging software ImageJ. Synaptic loss was analyzed by determining the thickness of stratum lucidum within the same hippocampal CA3 region used for NeuN quantification. Microglial and astrocytic cells per hippocampal section were quantified by counting the number of Iba1-positive or GFAP-positive cell bodies, respectively. For each mouse, three sections per brain and two visual fields (each $500 \mu \mathrm{m} \times 500 \mu \mathrm{m}$ ) per section were quantified. Quantification was done with ImageJ 1.48a software (National Institutes of Health, USA).

\section{Open-field test and object-place recognition}

Novelty recognition memory was tested in the context of an object-place recognition task. Only mice without obvious motor phenotype were tested in the object-place recognition task. Behavioral testing was performed as described by Bevins and Besheer [7] with minor modifications. Briefly, mice were handled for $1 \mathrm{~min}$ per day for 3 days. Then, mice were habituated to the testing environment and procedure for another 3 days. Therefore, mice were transferred to the testing room and given at least $1 \mathrm{~h}$ to acclimate. Mice were habituated to the arena (white $25 \times 25 \mathrm{~cm}$ square plastic chambers) without stimuli for $15 \mathrm{~min}$. The first habituation session was recorded for subsequent data analysis with an automated tracking system (Ethovision XT, Noldus Information Technology) and analyzed as an open-field test. On the forth day (training session), mice were allowed to explore the arena that now contained two identical objects (small glass bottles) in defined locations of the arena for a period of $10 \mathrm{~min}$. After a 1-h delay, the mice were presented with the same two objects and the test phase began. During testing, one object was in the exact same location as during the training phase, whereas the other object was placed in a novel location. The mice were allowed to explore the objects for $5 \mathrm{~min}$. The arena and objects were cleaned with $70 \%$ ethanol between trials to remove olfactory cues. Behavior was scored as object exploration, whenever the animal's nose was in contact with the object or directed 
towards the object (distance $\leq 2 \mathrm{~cm}$ ). Discrimination ratios were calculated as following: exploration time object 1 or $2 /$ sum of exploration times object 1 and 2 . Behavior was videotaped for offline analysis and an experienced observer quantified exploration times.

\section{Statistical analysis}

Quantifications and statistical analysis were carried out using GraphPad Prism. To determine statistically significance $t$ tests, two-way ANOVA tests, Mann-Whitney tests, and Log-Rank tests were carried out. The results of the significance tests are reported as follows: $* p<0.05$; $* * p<0.01 ; * * * p<0.001$. All values are displayed as mean \pm SEM if not otherwise stated.

\section{Results}

\section{Reduced tau aggregation in vitro}

First, we tested whether anle $138 \mathrm{~b}$ binds to aggregated tau protein in vitro. In a fluorescence spectroscopy assay, the fluorescence properties of anle $138 \mathrm{~b}$ at an excitation of $300 \mathrm{~nm}$ were analyzed after addition of recombinant tau protein. Anle138b exhibits weak fluorescence intensity in aqueous buffer, which is unchanged after addition of monomeric tau protein (Fig. 1a). After addition of in vitro aggregated tau the fluorescence intensity of anle $138 \mathrm{~b}$ increased, indicating a structure-dependent binding of anle138b to aggregated tau protein (Fig. 1a). In a cell free in vitro tau aggregation assay with subsequent scanning for intensely fluorescent targets (SIFT) analysis [8], we examined the effect of anle138b on tau aggregation (Fig. 1b). The tau aggregation assay is closely related to an assay that was used to study the effect of DPP compounds on alpha-synuclein aggregation [47] and the effect of tau phosphorylation on oligomer formation [35]. Since Castillo-Carranza [14] recently hypothesized that tau oligomers are the main toxic particle species in tauopathies, we analyzed the fluorescence data from the tau aggregation assay by cross-correlation (Fig. 1c) as cross-correlation detects oligomeric protein aggregates with higher sensitivity. We found that anle138b significantly suppressed tau oligomer formation (Fig. 1c). Next, we analyzed the tau anti-aggregative effect and the brain concentration of a couple of DPP compounds (Suppl. Fig. 1). Since anle138b offers the best correlation of in vitro tau aggregation inhibition and bioavailability (Fig. 1d), we chose anle138b for administration in PS19 mice. Thus, our data indicate that anle138b binds to aggregated tau protein and inhibits the formation of potentially toxic oligomeric tau aggregate species in vitro.

\section{Anle138b inhibits the formation of tau aggregates} in vivo

Since the brain bioavailability of many compounds is low due to insufficient blood-brain barrier penetration, we determined whether anle138b supplemented in food pellets effectively passes the blood-brain barrier. Treatment with anle138b-chow led to micromolar concentration of the compound in the brain (Suppl. Fig. 2). Since anle138b inhibited the formation of tau aggregates in vitro, we asked whether anle138b similarly affects tau aggregation in vivo. To determine the extent of tau pathology in the brains of 10- to 12-month-old mice treated from weaning to terminal phase of disease, we used multiple immunohistochemical and tangle specific stains to address the histopathological features of tau pathology (Fig. 2). First, we assessed the area of phosphorylated tau detected with the phosphorylation-specific tau antibody AT8 [21]. In ntg mice, no phosphorylated tau was detectable (Fig. 2a). In PS19 mice phosphorylated tau was detected in cortical areas like the hippocampus, entorhinal and piriform cortex. Anle138b reduced the AT8-positive tau deposits in PS19 mice when comparing to untreated mice (Fig. 2a). A quantitative analysis of AT8 staining revealed that anle138b-treated PS19 mice had significantly less ( $50 \%)$ AT8-positive area than untreated PS19 mice (Fig. 2d). In addition, to asses the number of neurons in different brain regions, we counted the number of AT8-positive neurons in the hippocampus, somatosensory/auditory cortex, and piriform/entorhinal cortex (Suppl. Fig. 3). Whereas, in the hippocampus and somatosensory/auditory cortex the number of AT8-positive neurons was reduced in anle138b-treated PS19 mice, there was no change in the piriform/entorhinal cortex. To confirm that anle138b reduces tau pathology in PS19 mice, we stained brain sections with the conformation-specific tau antibody MC1 that detects misfolded tau [27]. Accordingly, the anle138b-treated group exhibited only $50 \% \mathrm{MC} 1$ reactivity of the untreated ones (Fig. 2b, e). Finally, we assessed with the Gallyas silver staining method whether anle $138 \mathrm{~b}$ treatment influenced tau tangle formation (Fig. 2c). Indeed, anle138b reduced the formation of silver-positive tau tangles in the cerebral cortex as well as in the brain stem by $\sim 60 \%$ (Fig. 2f; Suppl. Fig. 4).

Subsequently, we examined the levels of total and insoluble tau in the brains of PS19 mice using multiple tau antibodies. Similar levels of total tau observed in treated and untreated mice using the tau antibodies HT7 and E178 exclude a treatment-induced suppression of tau expression (Fig. 3a, b). Quantification with phosphorylation-specific antibodies showed that the level of phosphorylated tau at the AT8 site (Ser202 + Thr205) is reduced, whereas the tau levels at the AT180 site (Thr231) are unaltered (Fig. 3c, d). To further evaluate, whether anle138b treatment affected 

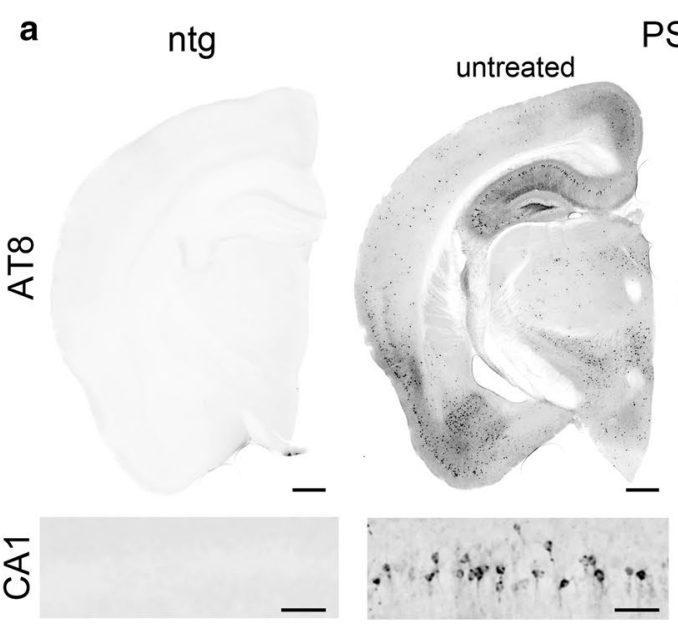

PS19
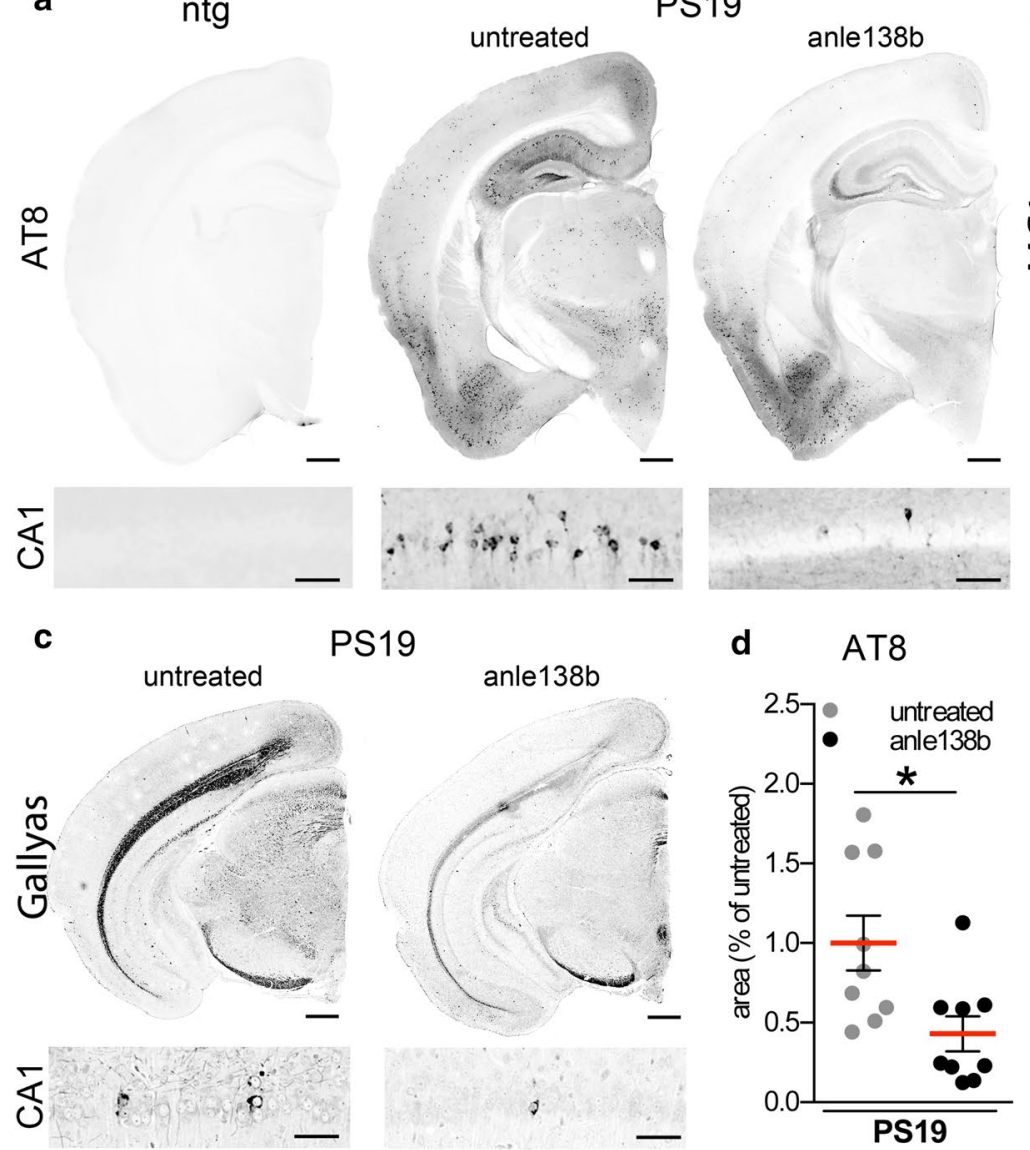

PS19

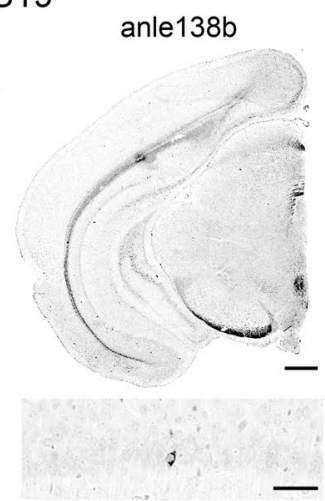

b

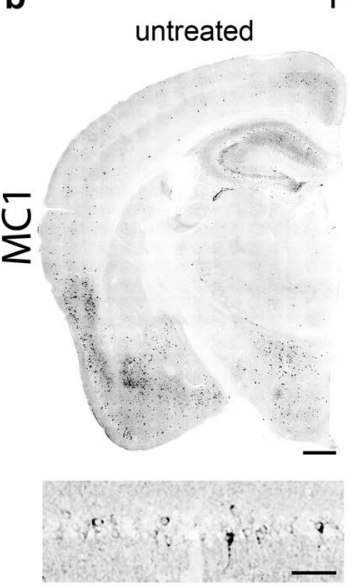

e
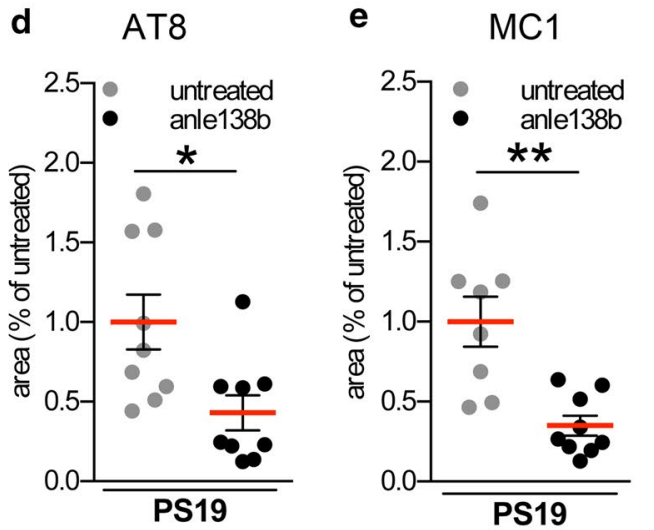

PS19

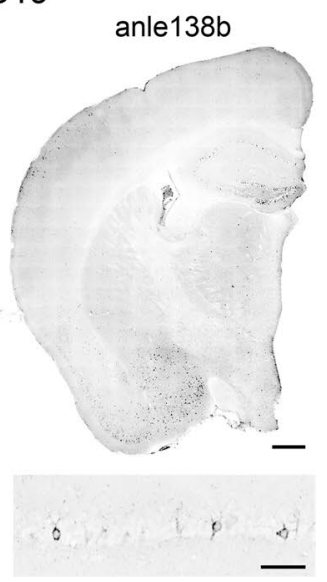

f

Gallyas

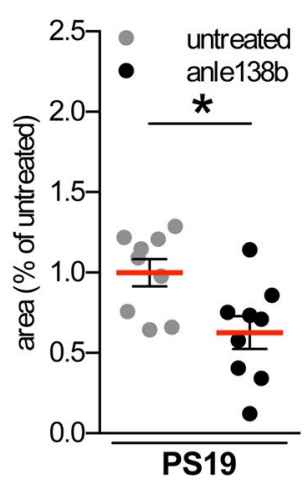

Fig. 2 Anle138b treatment reduces tau pathology in PS19 mice. a AT8 stainings of non-transgenic (ntg), untreated and anle138b-treated PS19 mice of coronal brain sections (upper panel) and hippocampal CA1 area (lower panel). b, c Immunohistochemical staining of brain sections with the conformation-specific tau antibody MC1 (b) and Gallyas silver staining (c) in treated and untreated PS19 mice. d-f Quantification of AT8, MC1 and silver-positive area in treated and untreated PS19 mice ( $n=9$ mice/group; $10-12$ months). Asterisks indicate significant differences relative to untreated PS19 mice $(* p<0.05 ; * * p<0.01 ; t$ test). Scale bars $500 \mu \mathrm{m}$ in low-power images and $50 \mu \mathrm{m}$ in high-power images

aggregates and significantly reduced larger insoluble tau species in the $50 \%$ sucrose fraction in relation to untreated animals (Fig. 3g, h). However, lower molecular weight tau aggregates (30-40\% sucrose) remained unchanged (Fig. 3g, h). Summarizing, the histological and biochemical data indicate that anle $138 \mathrm{~b}$ inhibits the formation of pathological higher molecular weight tau aggregates in vivo.

To examine whether the difference in the levels of tau phosphorylation upon anle138b treatment was due to changed kinase or phosphatase activity, we analyzed the effect on the tau kinase GSK3 and the tau phosphatase PP2A (Suppl. Fig. 5). The phosphorylation of GSK3 increased slightly but not significantly (Suppl. Fig. 5a), and neither the expression level of PP2A nor PP2A-activity was different upon anle138b incubation in vitro (Suppl. Fig. 5b, c). These results indicate that anle138b most probably does not exert its effect through changing kinase- or phosphatase-dependent tau phosphorylation status. Further (30-50\% sucrose) primarily contained insoluble $64 \mathrm{kD}$ tau (Fig. 3e). Anle138b altered the size distribution of tau 

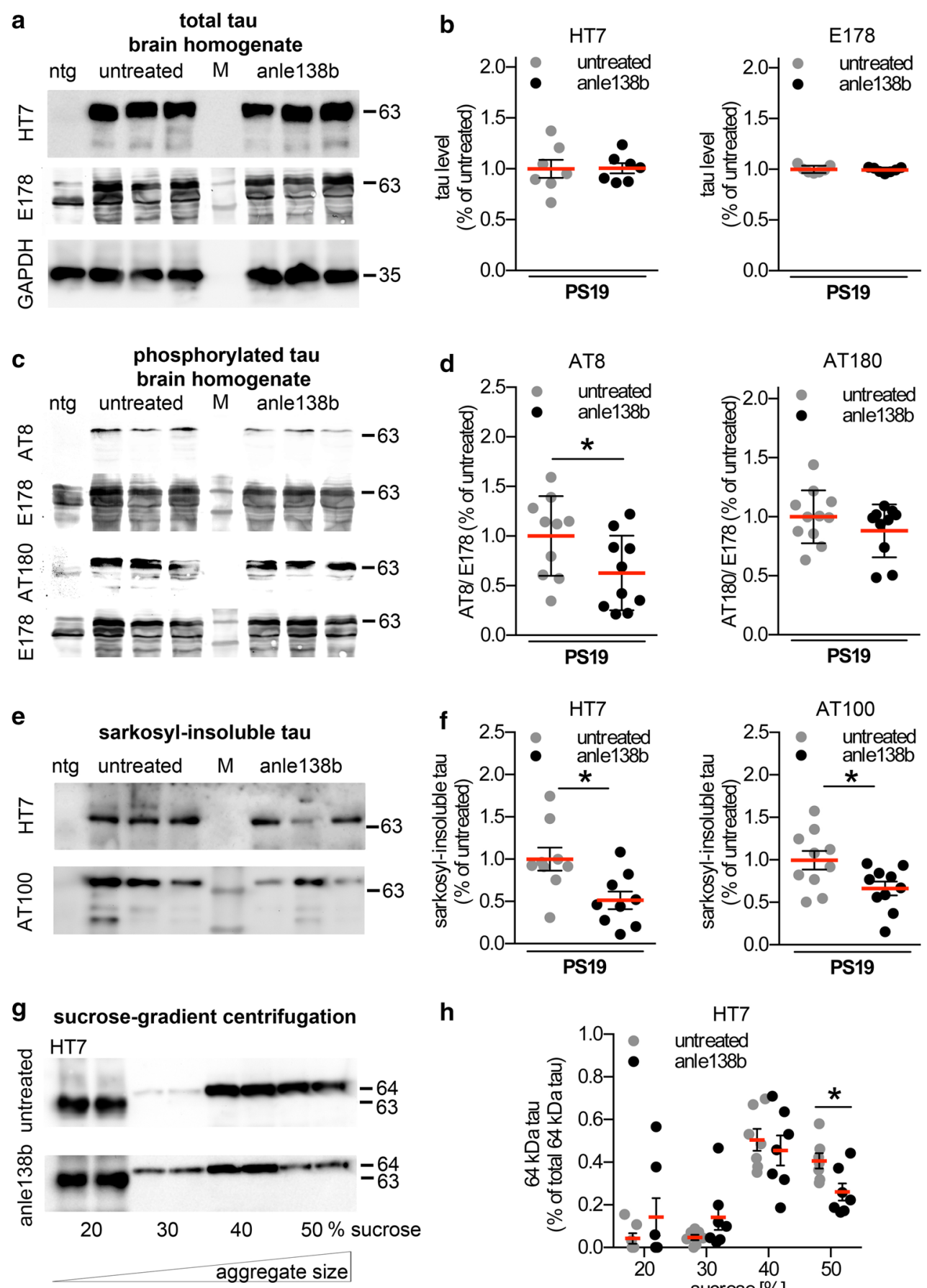

h

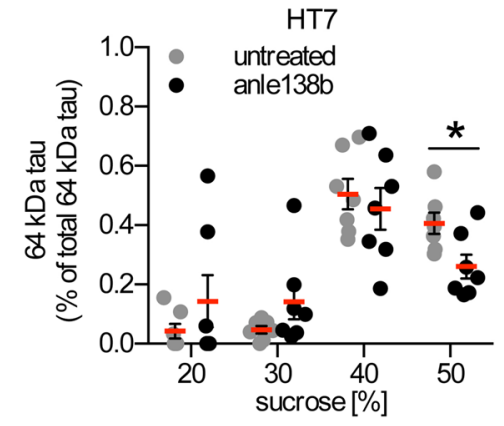

Fig. 3 Anle138b reduces insoluble tau levels in PS19 mice a Immunoblots of brain homogenates of non-transgenic (ntg), untreated and anle138b-treated PS19 mice probed with HT7 and E178 antibody detecting total tau. b Densitometric analysis of immunoblots showing the brain protein levels of total tau (HT7 and E178) in untreated and anle138b-treated PS19 mice. ( $n=7$ mice/group; 10-12 months). c Immunoblots of brain homogenates of ntg, untreated and anle138btreated PS19 mice probed with the phosphorylation-specific antibodies (AT8 and AT180) and phosphorylation-independent antibody E178. d Densitometric analysis of immunoblots showing phosphorylated tau (AT8, AT180) in untreated and anle138b-treated PS19 mice. ( $n=10-11$ mice/group; 10-12 months). Immunoblots using the anti-

bodies HT7 and AT100 (e) and densitometric analysis (f) of sarkosylinsoluble aggregated tau protein in the brain of untreated PS19 transgenic mice in comparison to anle138b-treated animals. $(n=9-10$ mice/group; 10-12 months). Immunoblots of sucrose-gradient centrifugation fractions from untreated and anle138b-treated mouse brain homogenates, to separate tau aggregates according to their molecular weight (g) and densitometric analysis (h). Anle138b-treated PS19 mice showed a significant reduction of high molecular weight species in the $50 \%$ sucrose fraction in comparison to untreated animals ( $n=7$ mice/group; 10-12 months). Asterisks indicate significant differences relative to untreated PS 19 mice $\left({ }^{*} p<0.05 ; t\right.$ test $)$ 
mechanisms for reduced tau pathology represent stimulated autophagy or increased protein degradation [16, 42]. Therefore, we examined the autophagy markers P62/SQSTM1 and LC3 in brains of 10- to 12-month-old PS19 mice as well as proteasomal tau degradation over time in anle138btreated neurons (Suppl. Fig. 5). After immunohistochemical staining, we found unchanged localization and intensity for P62/SQSTM1 and LC3 in both anle138b-treated and untreated PS19 mice (Suppl. Fig. 6a, b). As expected, P62/SQSTM1 and AT100-positive tau inclusions co-localized in PS19 mice (Suppl. Fig. 6a). For LC3, we detected unchanged immunoreactivity in brains of anle138btreated or untreated PS19 mice (Suppl. Fig. 6b). Immunoblots showed similar levels of P62/SQSTM1 and LC3 in anle138b-treated and untreated mice (Suppl. Fig. 6c). Densitometric analysis revealed that both autophagy markers were unchanged in anle138b-treated PS19 mice (Suppl. Fig. 6d, e). In primary neurons, anle138b treatment has no effect on proteasomal tau degradation and tau ubiquitination (Suppl. Fig. 6f, g). Thus, our data indicate that the reduced levels of aggregated tau in brains of anle138btreated PS19 mice are related to other mechanisms than increased autophagy or enhanced proteasomal degradation.

\section{Attenuated neuropathological impairments}

In Alzheimer's disease, tau pathology correlates with synapse and neuron loss [23, 33]. PS19 mice recapitulate synapse and neuron loss in the hippocampal CA3 region [50]. Therefore, we quantified the area of NeuN-positive neurons in this brain region at 10-12 months of age. NeuNimmunoreactivity was unchanged comparing anle138btreated with untreated ntg mice (Fig. 4a, b). In PS19 mice, anle138b treatment significantly ameliorated neuronal loss (Fig. 4a, b). Next, we analyzed synaptic density in the stratum lucidum adjacent to the CA3 region, by immunohistochemical quantification of the marker synaptophysin. Untreated and anle138b-treated ntg littermates exhibited unchanged synapse density. However, untreated PS19 mice lost $55 \%$ of synapses in stratum lucidum that was ameliorated by anle138b (Fig. 4c, d).

In human tauopathies and PS19 mice, gliosis correlates with the distribution of tau pathology [5, 26, 28, 46, 50]. Therefore, we analyzed micro- and astrogliosis in brains of untreated and anle138b-treated mice. Anle138b treatment significantly reduced the number of microglia in the hippocampus compared with untreated PS19 mice (Fig. 4e, f). We also found that anle138b diminished GFAP staining in the hippocampus and reduced the number of activated astrocytes in PS19 mice compared with untreated animals (Fig. 4g, h). In ntg mice, the densities of both cell types were unaltered after anle138b treatment (Fig. 4g, h). Thus, anle138b treatment attenuated neuron and synapse loss in CA3 and stratum lucidum of the hippocampus. Moreover, neuroinflammation was less pronounced upon anle $138 \mathrm{~b}$ treatment, reflected by both reduced micro- and astrogliosis.

\section{Prolonged survival and improved cognition}

The high bioavailability of anle138b (Suppl. Fig. 2) allowed treating mice with the drug provided in food pellets. This convenient way of drug application further enabled us to treat mice for a time-period up to 17 months. Non-transgenic mice tolerated such a long treatment very well, since they did not show any change in survival compared to untreated mice (Fig. 5a). Like FTDP-17 patients that carry a P301S tau mutation [12, 32], PS19 mice showed accelerated mortality and $80 \%$ of the mice died within 12 months (Fig. 5a). We tested the effect of anle138b on survival and recognition memory in PS19 animals. By 12 months of age, the absolute survival rate of anle138b-treated PS19 mice was 2.9 times higher than for untreated animals $(p<0.01$; survival rates: untreated $16 \%$; anle138b $46 \%$; Fig. 5). Moreover, anle138b significantly prolonged the mean survival time of PS19 mice by almost 6 weeks (Fig. 5a; Anle138b: $362 \pm 12$ days vs. untreated: $321 \pm 10$ days; $n=37$ mice/group; $p<0.05 t$ test). In addition, the rate of decline for mouse weight after onset of disease was significantly higher in untreated PS19 compared to anle138b-treated PS19 mice (Suppl. Fig. 7). Anle138b had no influence on survival of ntg animals (Fig. 5a). Thus, PS19 mice treated with anle138b showed an increased 12 months absolute survival rate and a prolonged mean survival time.

Finally, since PS19 mice show deficits in spatial learning and memory [11], we addressed whether anle138b treatment improved cognition. We carried out an object-place recognition task at 9 months of age and compared untreated and anle138b-treated animals (Fig. 5b). Ntg mice showed the expected increased exploration of an object in a novel location relative to an identical object in a known location, indicating that animals had successfully memorized object-place relationships in this task. In untreated PS19 animals, in contrast, exploration times of the object in the novel location did not differ significantly from those of the object in the known location, which is consistent with the notion of learning and memory impairments in PS19 mice [11]. Anle138b-treated PS19 animals, however, showed discrimination ratios that were restored to the levels of $n t g$ mice, indicating that anle138b improved cognitive function in PS19 mutants. An Open-field test showed that this effect was not based on a motor deficit, since both groups traveled similar distances (Fig. 5c). Anle138b did not influence behavioral measures in ntg mice in any obvious manner, excluding a cognitive enhancer effect of the compound. 

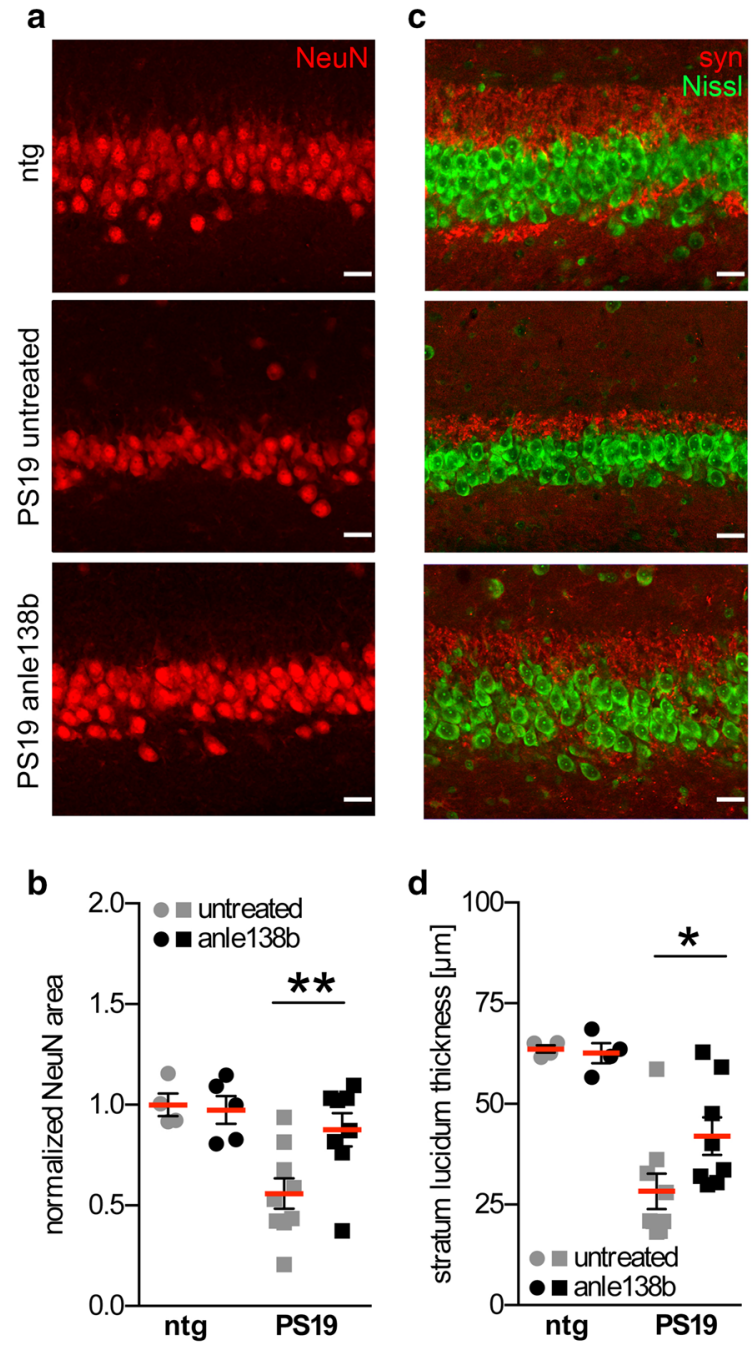

Fig. 4 Anle138b treatment ameliorates neuropathology of PS19 mice NeuN staining (a) and quantification (b) of the hippocampal CA3 region of ntg, untreated and anle138b-treated PS19 mice. Staining with the synaptic marker synaptophysin in the stratum lucidum (c) and quantification (d) of ntg, untreated and anle138b-treated PS19 mice. Microglia Iba1 and astrocyte GFAP staining (e, g) and

Together, these data indicate that anle $138 \mathrm{~b}$ treatment was sufficient to prevent the development of cognitive impairments in PS19 mice.

\section{Discussion}

We found that anle138b inhibits the formation of pathological tau aggregates in vitro and in vivo and thereby improves cognition, neuropathology and survival in a mouse model of tauopathies. Beyond that, anle138b represents a novel compound that significantly attenuates all features of human tauopathies recapitulated in the PS19 mouse model, such as cognitive decline, neuropathology, and accelerated
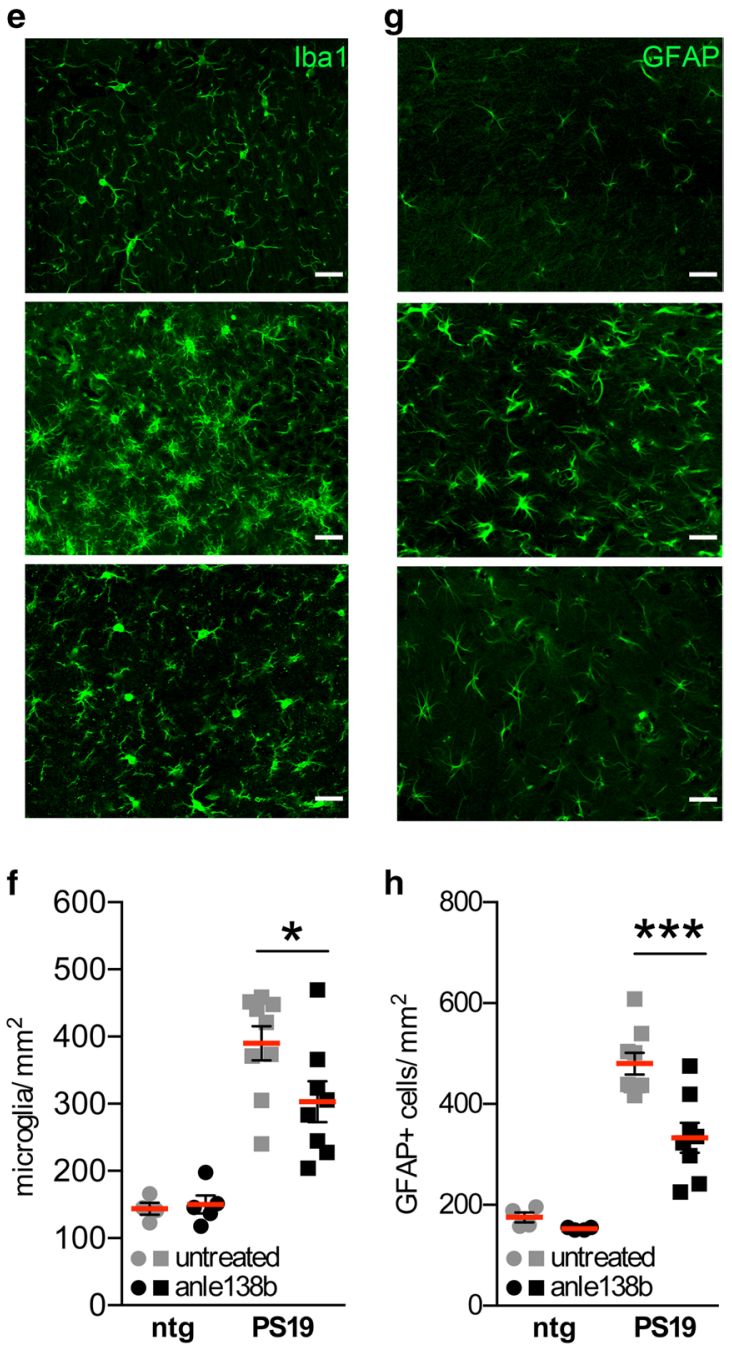

quantification (f, h) of ntg and PS19 mice treated and untreated with anle138b. a-h ( $n=8-9$ PS19 mice/group; $n=4-5$ ntg mice/group; 10-12 months). Scale bars $25 \mu \mathrm{m}$. Asterisks indicate significant differences relative to untreated PS19 mice $(* p<0.05 ; * * p<0.01$; $* * * p<0.001$; two-way ANOVA with Bonferroni's multiple-comparisons test)

mortality $[11,50]$. Remarkably, anle138b preserves learning and memory at a time, when untreated mice become terminally ill, which points to a longer disease-free survival accompanied with unimpaired cognition. Thus, our findings corroborate the concept that pathological tau aggregation plays a critical role in the pathogenesis of tauopathies [44] and pharmacological inhibition thereof represents a valid therapeutic target for a disease-modifying treatment of tauopathies.

The tau aggregation inhibitor methylene blue has been tested in tau transgenic mice as well [24, 48]. Methylene blue has multiple protein and molecular targets in vivo $[1,16,20,36,37]$, so that the observed effects may not be exclusively based on the prevention of tau aggregation 

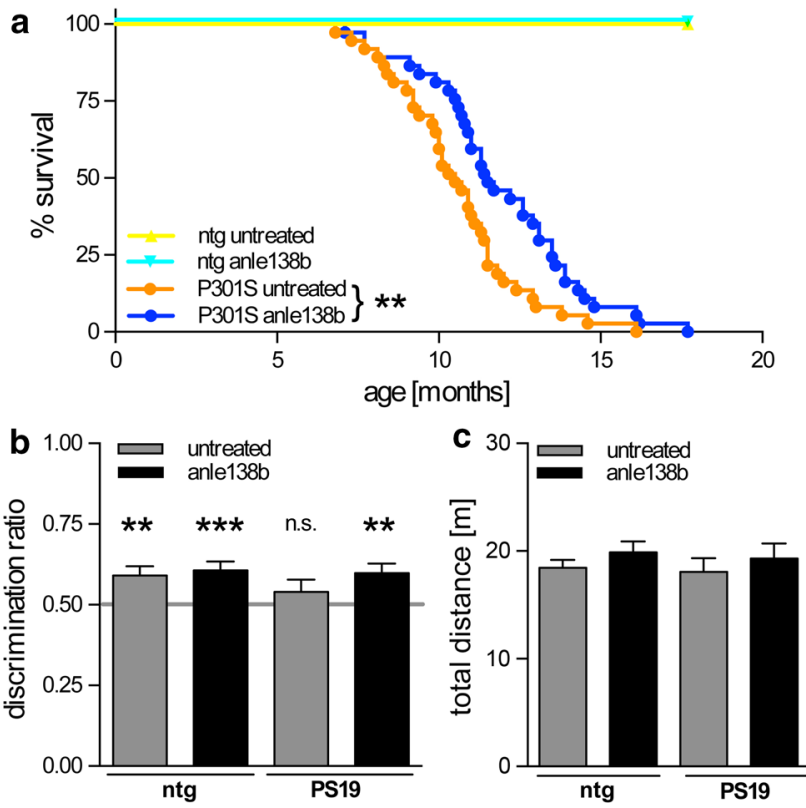

Fig. 5 Effect of anle138b treatment on survival and behavior in PS19 transgenic mice a Kaplan-Meier plots of PS19 transgenic and $\mathrm{ntg}$ mice. Anle $138 \mathrm{~b}$ treatment significantly prolonged survival time of PS19 mice $(* * p<0.01 ; n=37$ mice/group; Log-Rank test) and attenuated 12-month survival rate compared with untreated PS19 littermates $(p<0.01$, Log-Rank test). b Learning and memory were analyzed at 9 months of age using an object-place recognition task. Gray line indicates chance level. Untreated PS19 mice did not show significantly higher exploration of the object in the novel location relative to the object in the known location (discrimination ratio n.s.), which is consistent with memory impairments. In contrast, Anle138btreated PS19 mice spent significantly more time exploring the object in the novel location, indicating that treatment restored learning and memory in PS19 mice $(* * p<0.01 ; * * * p<0.001 ; n=19-24$ mice/ group; Significantly different from a hypothetical 0.50 , one-sample $t$ test). c Total distance traveled in the open-field test was similar in untreated and anle138b-treated mice independent of the genotype, indicating that motor behavior was unaffected in these animals ( $n=19-24$ mice/group)

[31]. However, anle138b seems to primarily act by directly targeting formation of pathological tau aggregates rather than by secondary off-target effects, since we found unchanged tau expression, tau ubiquitination and autophagy after administration of anle138b. With regard to the mode of action of anle138b, we showed that anle $138 \mathrm{~b}$ binds specifically to pre-aggregated tau and reduces the amount of oligomeric tau species at the molecular level in vitro. Upon anle138b treatment in PS19 mice, we detected reduced tau pathology in brain slices using histological stainings for phosphorylated and tangle tau. Biochemically, we found a reduced amount of large tau aggregate species without increasing the level of smaller tau aggregates in anle138b-treated PS19 mice. These results support the hypothesis that higher molecular weight aggregates and tangles are neurotoxic and suggest that the therapeutic effect of anle138b in PS19 mice is based on inhibiting the pathological aggregation process. In addition, there was neither an immune modulatory effect nor an obvious effect on cognition in ntg mice. Taken together, our data indicate that inhibition of tau aggregation is beneficial in vivo. Although further studies have to confirm the efficacy of anle $138 \mathrm{~b}$ before translation into human patients, we provide proof-ofprinciple that pharmacological inhibition of pathological tau aggregation represents a feasible treatment option for tauopathies.

In addition to tau aggregation inhibition, anle $138 \mathrm{~b}$ has been previously shown to inhibit prion protein and $\alpha$-synuclein aggregation [47]. These results point at a binding of anle $138 \mathrm{~b}$ to a common feature immanent of all three proteins.

Since tau pathology is associated with clinical symptoms in tauopathies [2, 10, 18], tau-directed therapies have moved into the focus of recent Alzheimer's disease research [17]. Recently, potential tau-directed therapeutic approaches involved immunotherapy [9, 15, 49], or microtubule-stabilizing drugs [11, 51], or enhanced autophagy $[16,42]$. Most of these studies successfully reduced tau pathology and improved behavior. However, none of these studies aimed at inhibiting the tau aggregation at the molecular level. Here, we present a tau aggregation inhibitor that ameliorates neuropathology and improves behavior. Notably, treatment up to 17 months in non-transgenic mice was well tolerated promising a high long-term therapy potential. Moreover, anle138b represents the first small molecule that significantly extended the mean survival time of tau transgenic mice.

Since none of the published tau-directed treatment strategies, including our study, were sufficient to cure the disease in tau transgenic mice, we suggest that future treatment strategies should be diversified to aim at various tau targets. It seems plausible that a combination of various therapeutic approaches like blocking the spread of tau pathology, stabilizing microtubules, enhancing autophagy, or inhibiting tau aggregation would be more effective.

In conclusion, inhibiting tau aggregation by small molecules represents a promising approach for the treatment of human tauopathies, where anle138b might serve as a new lead for developing a disease-modifying therapy.

Acknowledgments This work was supported by the German Center for Neurodegenerative Diseases (DZNE), the German research foundation (DFG; SFB1089 and KFO177 M.F.), Centers of Excellence in Neurodegeneration (CoEN; S. Re. and M. F.), European Union (MultiSyn to C. G. and A. G.), and the Max Planck Society (C. G.). We thank Peter Davies for kindly providing us with the MC1 antibody. We thank Gabor Petzold for helpful discussions on the manuscript. The authors J. W., S. Ry., A. L., H. A. K., C. G., and A. G. are inventors in a patent application related to the novel compound presented in this manuscript. AG and CG are shareholder of MODAG GmbH. 


\section{References}

1. Akoury E, Pickhardt M, Gajda M, Biernat J, Mandelkow E, Zweckstetter M (2013) Mechanistic basis of phenothiazinedriven inhibition of Tau aggregation. Angew Chem 52:35113515. doi:10.1002/anie. 201208290

2. Arriagada PV, Growdon JH, Hedley-Whyte ET, Hyman BT (1992) Neurofibrillary tangles but not senile plaques parallel duration and severity of Alzheimer's disease. Neurology 42:631-639

3. Bader B, Nubling G, Mehle A, Nobile S, Kretzschmar H, Giese A (2011) Single particle analysis of tau oligomer formation induced by metal ions and organic solvents. Biochem Biophys Res Commun 411:190-196. doi:10.1016/j.bbrc.2011.06.135

4. Ballatore C, Brunden KR, Piscitelli F et al (2010) Discovery of brain-penetrant, orally bioavailable aminothienopyridazine inhibitors of tau aggregation. J Med Chem 53:3739-3747. doi:10.1021/jm100138f

5. Bellucci A, Westwood AJ, Ingram E, Casamenti F, Goedert M, Spillantini MG (2004) Induction of inflammatory mediators and microglial activation in mice transgenic for mutant human P301S tau protein. Am J Pathol 165:1643-1652. doi:10.1016/ S0002-9440(10)63421-9

6. Berger Z, Roder H, Hanna A et al (2007) Accumulation of pathological tau species and memory loss in a conditional model of tauopathy. J Neurosci Off J Soc Neurosci 27:3650-3662. doi:10.1523/JNEUROSCI.0587-07.2007

7. Bevins RA, Besheer $\mathbf{J}$ (2006) Object recognition in rats and mice: a one-trial non-matching-to-sample learning task to study 'recognition memory'. Nat Protoc 1:1306-1311. doi:10.1038/ nprot.2006.205

8. Bieschke J, Giese A, Schulz-Schaeffer W et al (2000) Ultrasensitive detection of pathological prion protein aggregates by dualcolor scanning for intensely fluorescent targets. Proc Natl Acad Sci USA 97:5468-5473

9. Boutajangout A, Quartermain D, Sigurdsson EM (2010) Immunotherapy targeting pathological tau prevents cognitive decline in a new tangle mouse model. J Neurosci Off J Soc Neurosci 30:16559-16566. doi:10.1523/JNEUROSCI.4363-10.2010

10. Braak H, Braak E (1991) Neuropathological stageing of Alzheimer-related changes. Acta Neuropathol 82:239-259

11. Brunden KR, Zhang B, Carroll J et al (2010) Epothilone D improves microtubule density, axonal integrity, and cognition in a transgenic mouse model of tauopathy. J Neurosci Off J Soc Neurosci 30:13861-13866. doi:10.1523/JNEUROSCI.3059-10.2010

12. Bugiani O, Murrell JR, Giaccone G et al (1999) Frontotemporal dementia and corticobasal degeneration in a family with a P301S mutation in tau. J Neuropathol Exp Neurol 58:667-677

13. Bulic B, Pickhardt M, Khlistunova I et al (2007) Rhodaninebased tau aggregation inhibitors in cell models of tauopathy. Angew Chem 46:9215-9219. doi:10.1002/anie.200704051

14. Castillo-Carranza DL, Gerson JE, Sengupta U, Guerrero-Munoz MJ, Lasagna-Reeves CA, Kayed R (2014) Specific targeting of tau oligomers in Htau mice prevents cognitive impairment and tau toxicity following injection with brain-derived tau oligomeric seeds. J Alzheimers Dis 40(Suppl 1):S97-S111. doi:10.3233/ JAD-132477

15. Chai X, Wu S, Murray TK et al (2011) Passive immunization with anti-Tau antibodies in two transgenic models: reduction of Tau pathology and delay of disease progression. J Biol Chem 286:34457-34467. doi:10.1074/jbc.M111.229633

16. Congdon EE, Wu JW, Myeku N et al (2012) Methylthioninium chloride (methylene blue) induces autophagy and attenuates tauopathy in vitro and in vivo. Autophagy 8:609-622. doi:10.4161/auto.19048
17. Giacobini E, Gold G (2013) Alzheimer disease therapy-moving from amyloid-beta to tau. Nat Rev Neurol 9:677-686. doi:10.1038/nrneurol.2013.223

18. Giannakopoulos P, Herrmann FR, Bussiere T et al (2003) Tangle and neuron numbers, but not amyloid load, predict cognitive status in Alzheimer's disease. Neurology 60:1495-1500

19. Giese A, Levin J, Bertsch U, Kretzschmar H (2004) Effect of metal ions on de novo aggregation of full-length prion protein. Biochem Biophys Res Commun 320:1240-1246. doi:10.1016/j. bbrc.2004.06.075

20. Gillman PK (2011) CNS toxicity involving methylene blue: the exemplar for understanding and predicting drug interactions that precipitate serotonin toxicity. J Psychopharmacol 25:429-436. doi:10.1177/0269881109359098

21. Goedert M, Jakes R, Vanmechelen E (1995) Monoclonal antibody AT8 recognises tau protein phosphorylated at both serine 202 and threonine 205. Neurosci Lett 189:167-169

22. Gogolla N, Galimberti I, DePaola V, Caroni P (2006) Staining protocol for organotypic hippocampal slice cultures. Nat Protoc 1:2452-2456. doi:10.1038/nprot.2006.180

23. Gomez-Isla T, Hollister R, West H et al (1997) Neuronal loss correlates with but exceeds neurofibrillary tangles in Alzheimer's disease. Ann Neurol 41:17-24. doi:10.1002/ana.410410106

24. Hosokawa M, Arai T, Masuda-Suzukake M et al (2012) Methylene blue reduced abnormal tau accumulation in P301L tau transgenic mice. PLoS One 7:e52389. doi:10.1371/journal. pone. 0052389

25. Huang Y, Mucke L (2012) Alzheimer mechanisms and therapeutic strategies. Cell 148:1204-1222. doi:10.1016/j. cell.2012.02.040

26. Ishizawa K, Dickson DW (2001) Microglial activation parallels system degeneration in progressive supranuclear palsy and corticobasal degeneration. J Neuropathol Exp Neurol 60:647-657

27. Jicha GA, Bowser R, Kazam IG, Davies P (1997) Alz-50 and $\mathrm{MC}-1$, a new monoclonal antibody raised to paired helical filaments, recognize conformational epitopes on recombinant tau. J Neurosci Res 48:128-132

28. Kersaitis C, Halliday GM, Kril JJ (2004) Regional and cellular pathology in frontotemporal dementia: relationship to stage of disease in cases with and without Pick bodies. Acta Neuropathol 108:515-523. doi:10.1007/s00401-004-0917-0

29. Khlistunova I, Biernat J, Wang Y et al (2006) Inducible expression of Tau repeat domain in cell models of tauopathy: aggregation is toxic to cells but can be reversed by inhibitor drugs. J Biol Chem 281:1205-1214. doi:10.1074/jbc.M507753200

30. Lee VM, Goedert M, Trojanowski JQ (2001) Neurodegenerative tauopathies. Annu Rev Neurosci 24:1121-1159. doi:10.1146/ annurev.neuro.24.1.1121

31. Lee VM, Brunden KR, Hutton M, Trojanowski JQ (2011) Developing therapeutic approaches to tau, selected kinases, and related neuronal protein targets. Cold Spring Harb Perspect Med 1:a006437. doi:10.1101/cshperspect.a006437

32. Lossos A, Reches A, Gal A et al (2003) Frontotemporal dementia and parkinsonism with the P301S tau gene mutation in a Jewish family. J Neurol 250:733-740. doi:10.1007/s00415-003-1074-4

33. Masliah E (2001) Recent advances in the understanding of the role of synaptic proteins in Alzheimer's disease and other neurodegenerative disorders. J Alzheimers Dis 3:121-129

34. Masuda M, Suzuki N, Taniguchi S et al (2006) Small molecule inhibitors of alpha-synuclein filament assembly. Biochemistry 45:6085-6094. doi:10.1021/bi0600749

35. Nubling G, Bader B, Levin J, Hildebrandt J, Kretzschmar H, Giese A (2012) Synergistic influence of phosphorylation and metal ions on tau oligomer formation and coaggregation with alpha-synuclein at the single molecule level. Mol Neurodegener 7:35. doi:10.1186/1750-1326-7-35 
36. O'Leary JC 3rd, Li Q, Marinec P et al (2010) Phenothiazinemediated rescue of cognition in tau transgenic mice requires neuroprotection and reduced soluble tau burden. Mol Neurodegener 5:45. doi:10.1186/1750-1326-5-45

37. Oz M, Lorke DE, Hasan M, Petroianu GA (2011) Cellular and molecular actions of Methylene Blue in the nervous system. Med Res Rev 31:93-117. doi:10.1002/med.20177

38. Pickhardt M, Gazova Z, von Bergen M et al (2005) Anthraquinones inhibit tau aggregation and dissolve Alzheimer's paired helical filaments in vitro and in cells. J Biol Chem 280:3628 3635. doi:10.1074/jbc.M410984200

39. Pickhardt M, Larbig G, Khlistunova I et al (2007) Phenylthiazolyl-hydrazide and its derivatives are potent inhibitors of tau aggregation and toxicity in vitro and in cells. Biochemistry 46:10016-10023. doi:10.1021/bi700878g

40. Polydoro M, Acker CM, Duff K, Castillo PE, Davies P (2009) Age-dependent impairment of cognitive and synaptic function in the htau mouse model of tau pathology. J Neurosci Off J Soc Neurosci 29:10741-10749. doi:10.1523/ JNEUROSCI.1065-09.2009

41. Santacruz K, Lewis J, Spires T et al (2005) Tau suppression in a neurodegenerative mouse model improves memory function. Science 309:476-481. doi:10.1126/science.1113694

42. Schaeffer V, Lavenir I, Ozcelik S, Tolnay M, Winkler DT, Goedert M (2012) Stimulation of autophagy reduces neurodegeneration in a mouse model of human tauopathy. Brain J Neurol 135:2169-2177. doi:10.1093/brain/aws143

43. Sperfeld AD, Collatz MB, Baier H et al (1999) FTDP-17: an early-onset phenotype with parkinsonism and epileptic seizures caused by a novel mutation. Ann Neurol 46:708-715

44. Spillantini MG, Goedert M (2013) Tau pathology and neurodegeneration. Lancet Neurol 12:609-622. doi:10.1016/ S1474-4422(13)70090-5
45. Terwel D, Lasrado R, Snauwaert J et al (2005) Changed conformation of mutant Tau-P301L underlies the moribund tauopathy, absent in progressive, nonlethal axonopathy of Tau- $4 \mathrm{R} / 2 \mathrm{~N}$ transgenic mice. J Biol Chem 280:3963-3973. doi:10.1074/jbc. M409876200

46. Togo T, Dickson DW (2002) Tau accumulation in astrocytes in progressive supranuclear palsy is a degenerative rather than a reactive process. Acta Neuropathol 104:398-402. doi:10.1007/ s00401-002-0569-x

47. Wagner J, Ryazanov S, Leonov A et al (2013) Anle138b: a novel oligomer modulator for disease-modifying therapy of neurodegenerative diseases such as prion and Parkinson's disease. Acta Neuropathol 125:795-813. doi:10.1007/s00401-013-1114-9

48. Wischik CM, Edwards PC, Lai RY, Roth M, Harrington CR (1996) Selective inhibition of Alzheimer disease-like tau aggregation by phenothiazines. Proc Natl Acad Sci USA 93:11213-11218

49. Yanamandra K, Kfoury N, Jiang H et al (2013) Anti-tau antibodies that block tau aggregate seeding in vitro markedly decrease pathology and improve cognition in vivo. Neuron 80:402-414. doi:10.1016/j.neuron.2013.07.046

50. Yoshiyama Y, Higuchi M, Zhang B et al (2007) Synapse loss and microglial activation precede tangles in a P301S tauopathy mouse model. Neuron 53:337-351. doi:10.1016/j. neuron.2007.01.010

51. Zhang B, Carroll J, Trojanowski JQ et al (2012) The microtubule-stabilizing agent, epothilone $\mathrm{D}$, reduces axonal dysfunction, neurotoxicity, cognitive deficits, and Alzheimer-like pathology in an interventional study with aged tau transgenic mice. J Neurosci Off J Soc Neurosci 32:3601-3611. doi:10.1523/ JNEUROSCI.4922-11.2012 